\title{
Erratum to: Genetic diversity, structure and conservation of the endangered Cantabrian Capercaillie in a unique peripheral habitat
}

Fernando Alda - Manuel A. González • Pedro P. Olea • Vicente Ena • Raquel Godinho - Sergei V. Drovetski

Published online: 30 May 2013

(C) Springer-Verlag Berlin Heidelberg 2013

\section{Erratum to: Eur J Wildl Res}

$$
\text { DOI 10.1007/s10344-013-0727-6 }
$$

The original version of this article inadvertently contained a mistake. An incorrect version of Figure 4 was published. The correct figure appears below.

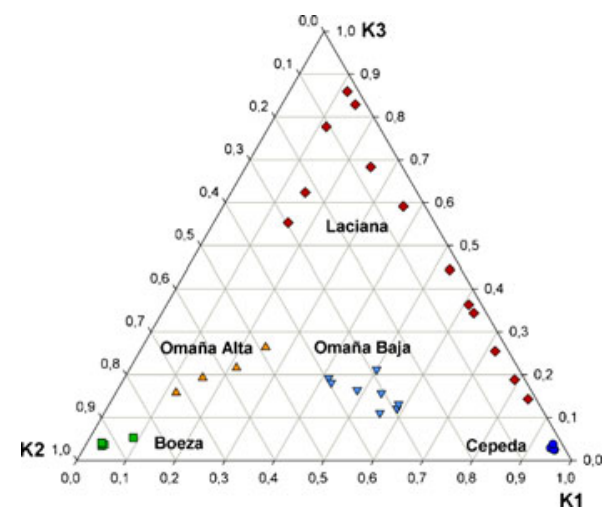

Fig. 4 Triangular plot representing individual assignment probabilities for each of the genetic groups inferred in STRUCTURE $(K=3)$. Each triangle corner represents an assignment probability $(q)$ of 1.0 for each of the inferred genetic groups

The online version of the original article can be found at http://dx.doi.org/ 10.1007/s10344-013-0727-6.

F. Alda $\cdot$ P. P. Olea

Instituto de Investigación en Recursos Cinegéticos (IREC, CSIC-UCLM-JCCM), Ronda de Toledo s/n, 13005 Ciudad Real, Spain

\section{F. Alda}

Smithsonian Tropical Research Institute, Apartado, 0843-03092 Balboa, Ancón, Republic of Panama

\section{A. González $(\bowtie) \cdot$ V. Ena}

Dpto. de Biodiversidad y Gestión Ambiental, Universidad de León, Campus de Vegazana s/n, 24071 León, Spain e-mail: magong@unileon.es
R. Godinho $\cdot$ S. V. Drovetski

CIBIO, Centro de Investigação em Biodiversidade e Recursos Genéticos, Campus Agrário de Vairão, Rua Padre Armando Quintas, Crasto, 4485-661 Vairão, Portugal

S. V. Drovetski

Tromsø University Museum, NO-9037 Tromsø, Norway 